\title{
Collective Thermotaxis of Thermally Active Colloids
}

\author{
Ramin Golestanian* \\ Rudolf Peierls Centre for Theoretical Physics, University of Oxford, Oxford OX1 3NP, UK
}

(Dated: June 7, 2018)

\begin{abstract}
Colloids with patchy metal coating under laser irradiation could act as local sources of heat due to the absorption of light. While for asymmetric colloids this could induce self-propulsion, it also leads to the generation of a slowly decaying temperature profile that other colloids could interact with. The collective behavior of a dilute solution of such thermally active particles is studied using a stochastic formulation. It is found that when the Soret coefficient is positive, the system could be described in stationary-state by the nonlinear Poisson-Boltzmann equation and could adopt density profiles with significant depletion in the middle region when confined. For colloids with negative Soret coefficient, the system can be described as a dissipative equivalent of a gravitational system. It is shown that in this case the thermally active colloidal solution could undergo an instability at a critical laser intensity, which has similarities to supernova explosion.
\end{abstract}

PACS numbers: 82.70.Dd,05.70.Ln,47.57.-s,47.70.-n

The motion of colloidal particles in a solution in the presence of an externally applied temperature gradient, which is known as thermophoresis or the Soret effect [1], has been studied since the $19^{\text {th }}$ century and observed in a variety of systems [2]. Although its existence can be well formulated in non-equilibrium thermodynamics within linear response theory [3], many aspects of the microscopic nature of the phenomenon has remained a subject of investigation to date [4]. The effect has also been shown to provide a powerful tool for manipulating macromolecules and colloids [5]. Since in the phoretic transport mechanisms the colloids experience no net force, it is possible to take advantage of them to design selfpropelled particles by incorporating a built-in mechanism that provides asymmetric sources that could generate and maintain the necessary gradient across them needed for propulsion [6, 7]. Recently, Jiang et al. 8] have shown that silica beads half-coated with gold when irradiated with a defocused laser beam exhibit such a propulsion, as the gold caps act as heat sources when they absorb light. Moreover, even without the self-propulsion, laser-heated gold-coated colloids have been shown to undergo substantially enhanced Brownian diffusion, which is related to the modification of the temperature in the medium and the resulting changes in the viscosity [9]. Since such thermally active colloids would create temperature profiles around them that decay as $1 / r$, in addition to causing them to self-propel thermophoresis could provide a mechanism for them to interact with one another in a solution. The long-ranged nature of the inter-colloidal thermophoretic interaction could lead to interesting collective behaviors.

Here we construct a stochastic formulation to describe the collective behavior of a number of thermally active colloids. At the long time and large length scale limit and for dilute solutions, the formulation simplifies to a set of two nonlinear coupled differential equations for the density and temperature profiles in the medium. In stationary-state, we provide a number of examples for which the equations could be solved exactly. They show a depletion effect for the case of positive Soret coefficient, and an instability at a finite laser intensity for negative Soret coefficient.

We consider $N$ colloidal particles of radius $R$ that are half-coated with a metal that absorbs the laser light with an efficiency $\epsilon$, thus creating a local source of heat of magnitude $\epsilon I$, where $I$ is the intensity of the laser (see Fig. 1). We assume that the laser intensity is uniform throughout the space, and thus ignore any optical confinement effect. In an externally generated temperature gradient, the colloids move with a drift velocity $\mathbf{v}=-D_{T} \nabla T$, where $D_{T}$ is the thermodiffusion coefficient. The asymmetric heat generation around each colloid provides a mechanism to create and maintain a local temperature gradient that leads to propulsion via self-thermophoresis 7]. For a Janus-sphere colloid, the propulsion velocity can be calculated as $v_{0}=\epsilon I D_{T} /(6 \kappa)$, where $\kappa$ is the thermal conductivity of the medium [7, 8, 10]. The stochastic motion of the $i$-th colloid is described by its

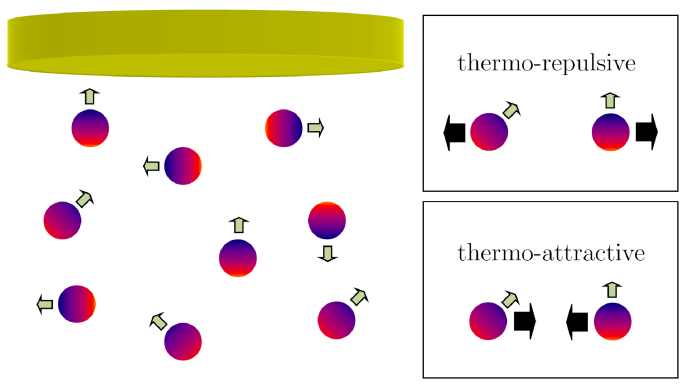

FIG. 1: (color online.) The metal-coated Janus-spheres under irradiation could self-propel in the directions shown by the (green) arrows and interact with one-another via the longranged temperature profiles they generate. The interactions are mutually repulsive when $S_{T}>0$ and attractive when $S_{T}<0$. 
instantaneous position $\mathbf{r}_{i}(t)$ and orientation $\mathbf{n}_{i}(t)$ that is a unit vector. They satisfy the Langevin equations $\frac{d \mathbf{r}_{i}}{d t}=v_{0} \mathbf{n}_{i}-D_{T} \nabla T\left(\mathbf{r}_{i}\right)+\boldsymbol{\xi}_{i}$ and $\frac{d \mathbf{n}_{i}}{d t}=\boldsymbol{\eta}_{i} \times \mathbf{n}_{i}$, in which $\boldsymbol{\xi}_{i}$ and $\boldsymbol{\eta}_{i}$ are Gaussian-distributed noise terms. Since each colloid generates heat with an overall (surfaceaverage) rate of $\frac{1}{2} \epsilon I$, the temperature profile at the location of each colloid is affected by the heat generated by all the other colloids. Since the temperature profile equilibrates considerably faster than the colloids, we have $T(\mathbf{r}, t)=T_{0}+\frac{\epsilon I R^{2}}{2 \kappa} \sum_{j} \frac{1}{\left|\mathbf{r}-\mathbf{r}_{j}(t)\right|}$, to the lowest order in the multipole expansion of the heat source distribution. While this approximation should be valid for sufficiently dilute colloidal solutions, the contributions from higher multipoles could readily be added to the above temperature profile. To probe the colloidal activity at shorter times than the rotational diffusion time, we need to incorporate the time dependence of heat diffusion, which could lead to anomalous dynamics of the colloid [11].

The Fokker-Planck equation for the probability distribution $\mathcal{P}(\mathbf{r}, \mathbf{n}, t) \equiv\left\langle\sum_{i=1}^{N} \delta\left(\mathbf{r}-\mathbf{r}_{i}(t)\right) \delta\left(\mathbf{n}-\mathbf{n}_{i}(t)\right)\right\rangle$, can be constructed from the Langevin equations as

$$
\partial_{t} \mathcal{P}+\nabla \cdot\left[v_{0} \mathbf{n} \mathcal{P}-D_{T}(\nabla T) \mathcal{P}-D \nabla \mathcal{P}\right]-D_{r} \mathcal{R}^{2} \mathcal{P}=0,
$$

where $\mathcal{R} \equiv \mathbf{n} \times \partial_{\mathbf{n}}$. In Eq. (11), $D$ and $D_{r}$ are the translational and rotational diffusion coefficients, respectively, and represent the corresponding widths of the Gaussian probability distributions for the noise terms $\boldsymbol{\xi}_{i}$ and $\boldsymbol{\eta}_{i}$ in the Langevin equations. In a medium with uniform temperature $T$, we have $D=k_{\mathrm{B}} T /(6 \pi \eta R)$ and $D_{r}=k_{\mathrm{B}} T /\left(8 \pi \eta R^{3}\right)$, where $\eta$ is the viscosity of water. Equation (11) should be complemented with the heat diffusion equation

$$
-\nabla^{2} T=\frac{2 \pi \epsilon I R^{2}}{\kappa} \int_{\mathbf{n}} \mathcal{P}(\mathbf{r}, \mathbf{n}),
$$

which describes how the temperature profile is affected by the spatial distribution of the colloids due to their role as motile heat sources. Equations (11) and (2) should be self-consistently solved to obtained the probability distribution of the colloids as well as the temperature profile in the medium.

Let us define the density $\rho(\mathbf{r})=\int_{\mathbf{n}} \mathcal{P}(\mathbf{r}, \mathbf{n})$, the polarization field $\mathbf{p}(\mathbf{r})=\int_{\mathbf{n}} \mathbf{n} \mathcal{P}(\mathbf{r}, \mathbf{n})$, and the nematic order parameter $\mathbf{Q}(\mathbf{r})=\int_{\mathbf{n}}^{\mathbf{n}}\left[\mathbf{n n}-\frac{1}{3} \mathbf{I}\right] \mathcal{P}(\mathbf{r}, \mathbf{n})$. Performing $\int_{\mathbf{n}}$ on Eq. (11), we can obtain an equation for the density as $\partial_{t} \rho+v_{0} \nabla \cdot \mathbf{p}-\nabla \cdot\left[D_{T}(\nabla T) \rho+D \nabla \rho\right]=0$, which is incomplete since it has a source term in the form of $-v_{0} \nabla \cdot \mathbf{p}$, which is present due to the self-propulsion of the colloids. Performing $\int_{\mathbf{n}} \mathbf{n} \times$ Eq. (1), we can obtain an equation for the polarization field as

$$
\begin{aligned}
& \partial_{t} \mathbf{p}+2 D_{r} \mathbf{p}+\frac{v_{0}}{3} \nabla \rho+v_{0} \nabla \cdot \mathbf{Q}(\mathbf{r}) \\
& -\nabla \cdot\left[D_{T}(\nabla T) \mathbf{p}+D \nabla \mathbf{p}\right]=0,
\end{aligned}
$$

where $\mathcal{R}^{2} \mathbf{n}=-2 \mathbf{n}$ is used. Equation (3) is also incomplete as it depends on $\mathbf{Q}$, and this hierarchy will continue for higher order cumulants.

To make further progress, we can seek to truncate the hierarchy and simplify Eq. (3) in some approximation. At time scales much longer than $1 / D_{r}$, the time derivative term in Eq. (3) is considerably smaller than $2 D_{r} \mathbf{p}$ and can thus be ignored. For sufficiently dilute solutions, i.e. when $\rho R^{3} \ll 1$, and in the absence of any external means that could induce polarization, such as external magnetic field for particles with a magnetic dipole moment or gravity [12, 13], we can ignore the $\nabla \cdot \mathbf{Q}(\mathbf{r})$ term. Since the time scale is much longer $1 / D_{r}$, any transient or initial ordering would also have decayed. Finally, at length scales much larger than $R$, the gradient terms in Eq. (3) can be neglected, and the equation can be approximated as

$$
\mathbf{p} \simeq-\frac{v_{0}}{6 D_{r}} \nabla \rho .
$$

Using Eq. (44), we can estimate that $\mathbf{Q} \sim \nabla \rho \nabla \rho$, ignoring which is consistent with our assumption of a dilute solution. Putting Eq. (4) back in the density equation, we find

$$
\partial_{t} \rho-\nabla \cdot\left[D_{\mathrm{eff}} \nabla \rho+D_{T}(\nabla T) \rho\right]=0,
$$

where $D_{\text {eff }}=D+v_{0}^{2} /\left(6 D_{r}\right)$ is the enhanced effective diffusion coefficient for the self-propelled active colloid [6, 11], which could also be rewritten as $D_{\text {eff }}=D\left[1+\frac{2}{9} \mathrm{Pe}^{2}\right]$ (for a sphere) in terms of the Peclet number $\mathrm{Pe}=v_{0} R / D$ [12]. Equation (5) should be solved in conjunction with the heat diffusion equation, which reads $-\nabla^{2} T=\frac{2 \pi \epsilon I R^{2}}{\kappa} \rho$.

In stationary state, Eq. (15) is satisfied if $D_{\text {eff }}^{\kappa} \nabla \rho+$ $D_{T}(\nabla T) \rho=0$, which can be written as $\nabla \ln \rho=$ $-\frac{D_{T}}{D_{\text {eff }}} \nabla T$. If we ignore the temperature dependence in $D_{\text {eff }}$, and use the Soret coefficient $S_{T}=D_{T} / D$, this can be integrated to yield

$$
\rho(\mathbf{r})=\rho_{0} \exp \left\{-\frac{S_{T}\left[T(\mathbf{r})-T_{0}\right]}{\left(1+\frac{2}{9} \mathrm{Pe}^{2}\right)}\right\} .
$$

Putting the above equation back in the heat diffusion equation yields a single nonlinear equation for the temperature profile as

$$
-\nabla^{2} T=\frac{2 \pi \epsilon I R^{2} \rho_{0}}{\kappa} \exp \left\{-\frac{S_{T}\left[T(\mathbf{r})-T_{0}\right]}{\left(1+\frac{2}{9} \mathrm{Pe}^{2}\right)}\right\} .
$$

Equation (7), which is reminiscent of the PoissonBoltzmann equation for electrolytes (see below), could be solved for the temperature profile, which then yields the stationary-state density profile of the colloids via Eq. (6). Note that the Soret coefficient could be both positive and negative. 
We can define an appropriate dimensionless temperature as $\Psi \equiv \frac{\left|S_{T}\right|\left[T(\mathbf{r})-T_{0}\right]}{1+\frac{2}{9} \mathrm{Pe}^{2}}$, and a characteristic length scale

$$
\ell \equiv \frac{\epsilon I R^{2}\left|S_{T}\right|}{2 \kappa\left(1+\frac{2}{9} \mathrm{Pe}^{2}\right)}
$$

which is reminiscent of the Bjerrum length in the electrostatic analogy. We have

$$
-\nabla^{2} \Psi=k^{2} e^{\mp \Psi}
$$

where $k^{2}=4 \pi \ell \rho_{0}$, and the sign choice is $-\operatorname{sgn}\left(S_{T}\right)$. Equation (9) is subject to the constraint $N=\rho_{0} \int_{\mathbf{r}} e^{\mp \Psi}$.

We now discuss a number of interesting exact solutions for the stationary-state described by Eq. (9). We consider colloidal solutions that are confined in some region in space and examine the effect of the dimensionality of the confinement geometry as well as the nature and the strength of the thermotactic coupling. The confinement could in practice come from the trapping effect of nonuniform laser beams, which could provide a very powerful tuning parameter. For simplicity, we model the confinement by introducing sharp boundaries such as confining walls.

When $S_{T}>0$, the electrostatic analogy is complete as the colloidal particles mutually repel one another, and the overall heat flux coming out of the solution through the boundaries of the confining "cage" is reminiscent of the electric field flux lines, which could be thought of as an outer shell of opposite charges maintaining neutrality and stability. We denote the $S_{T}>0$ case as thermorepulsive. This problem can be solved exactly for $1 \mathrm{D}$ and $2 \mathrm{D}$ confinements, and numerically for the 3D case [15]. When the colloidal solution is confined between two plates of lateral size $L$ and distance $2 h$, the density profile of the colloids is given as

$$
\rho(x)=\frac{\rho_{0}}{\left[1+\frac{2 \pi^{2} \ell^{2}}{k^{2}}\left(\frac{N}{L^{2}}\right)^{2}\right] \cos ^{2}\left(\frac{k x}{\sqrt{2}}\right)},
$$

where $\rho_{0}$ is the concentration at the edge of confinement (wall), and $k$ satisfies $\left(\frac{k h}{\sqrt{2}}\right) \tan \left(\frac{k h}{\sqrt{2}}\right)=\pi \ell h\left(\frac{N}{L^{2}}\right)$. The profile of Eq. (10) describes an accumulation of the colloids near the confining boundary (that is reminiscent to counterion condensation [14]) and a corresponding depletion of the central region of the system. In the strong coupling limit when $N \ell h / L^{2} \gg 1$, we can find an approximate solution to the transcendental equation as $k h \simeq \frac{\pi}{\sqrt{2}}\left[1-\frac{1}{\pi\left(N \ell h / L^{2}\right)}\right]$. In this limit, the ratio between the density of the colloids in the middle and at the edge can be found as $\frac{\rho_{\mathrm{m}}}{\rho_{0}} \simeq \frac{1}{4}\left(N \ell h / L^{2}\right)^{-2}$, which shows a significant depletion effect. Note that the depletion becomes stronger as $h$ is increased, when other parameters are kept fixed. The length $1 /\left[2 \pi \ell\left(N / L^{2}\right)\right]$ is equivalent to the Gouy-Chapman length in the electrostatic analogy

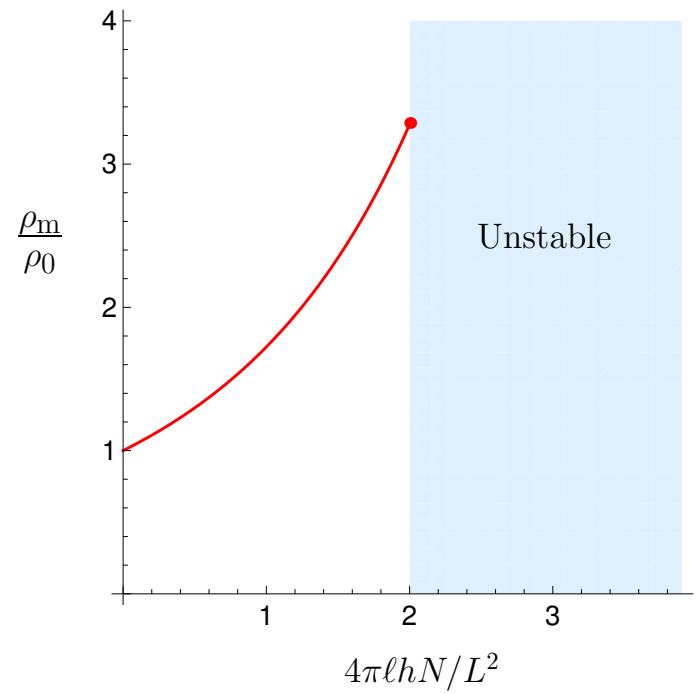

FIG. 2: (color online.) The density of colloids in the middle of the confined space relative to the density at the edge as a function of the dimensionless thermophoretic coupling constant for the thermo-attractive $\left(S_{T}<0\right)$ case. The critical density at the onset of instability is $\left(\rho_{\mathrm{m}} / \rho_{0}\right)_{c}=3.29$, which occurs at $\left(4 \pi \ell h N / L^{2}\right)_{c}=2$.

14]. For a colloidal solution trapped in a cylindrical cage of length $L$ and width $2 h$, the density profile reads

$$
\rho(r)=\frac{\rho_{0}}{\left[1+\frac{1}{2}\left(\frac{N \ell}{L}\right)\right]^{2}\left[1-\frac{1}{8} k^{2} r^{2}\right]^{2}},
$$

where $k h=\sqrt{\frac{8(N \ell / L)}{2+(N \ell / L)}}$. The strong coupling limit in this geometry corresponds to $N \ell / L \gg 1$, in which case we have $\frac{\rho_{\mathrm{m}}}{\rho_{0}} \simeq 4(N \ell / L)^{-2}$. Note that the magnitude of depletion is independent of the confinement size in this geometry. The ratio $\ell N / L$ is analogous to the socalled Manning-Oosawa parameter for highly charged rodlike polyelectrolytes [14]. A similar profile can be found when the colloidal solution is confined to a spherical cage of diameter $2 h$, where in the strong coupling limit that corresponds to $N \ell / h \gg 1$ in this case, we have $\frac{\rho_{\mathrm{m}}}{\rho_{0}} \simeq 21.4(N \ell / h)^{-2}$. Here, the depletion is inversely related to the size of the cage, namely it decreases for larger confinement sizes.

When $S_{T}<0$, the colloids attract each other and the problem is analogous to a gravitational system. We thus denote this case as thermo-attractive. Let us go back to the 1D confinement geometry, where the relevant thermophoretic coupling constant is $N \ell h / L^{2}$ as discussed above. In this case, Eq. (9) (with the positive sign choice) can be integrated in closed form and the density profile can be calculated. The stationary-state density profile (not presented here for brevity) shows that the particles will accumulate towards the center of the confined area. Figure 2 shows that the ratio between the density in the middle and at the edge of the confinement region increases as the thermophoretic coupling 
constant increases, up to a critical point beyond which a stable (stationary-state) solution no longer exists. The onset of instability occurs at $\left(4 \pi \ell h N / L^{2}\right)_{c}=2$, at which $\left(\rho_{\mathrm{m}} / \rho_{0}\right)_{c}=3.29$. Similar instabilities exist in the $2 \mathrm{D}$ and $3 \mathrm{D}$ confinement cases [16], where $N \ell / L$ and $N \ell / h$ play the role of the thermophoretic coupling constant, respectively.

The instability occurs because the particles that act as heat sources attract each other and could result in a suspension that becomes increasingly denser and hotter. In this case, the heat flux at the outer boundary of the system cannot balance the heat generated inside the confined region, which leads to an uncontrolled buildup of thermal energy. In molecular systems, an equation of the form of Eq. (7) (with $S_{T}<0$ ) is used to describe exothermic combustion reactions that could lead to thermal explosion [16]. It is not possible to predict what happens in our colloidal system above the onset of this instability using the present formulation, as the approximations used in its derivation are no longer valid. Within our handwaving analogy to a gravitational system, this explosion would have similarities to a type I supernova for a white dwarf, for which accreted material from the surroundings accelerates exothermic nuclear reaction to the point that the system becomes unstable [17]. This analogy is very rough, however, as the colloidal system operates in the dissipative regime as opposed to the inertial and conserved dynamics of the gravitational system.

We can estimate the length scale $\ell$ that characterizes the strength of thermophoretic interactions from the experiment of Ref. [8]. For $R=1 \mu \mathrm{m}$, we can estimate that for a fully coated bead that is not self-propelled due to lack of asymmetry $(\mathrm{Pe}=0), \ell \sim 10 \mu \mathrm{m}$, while for the self-propelled colloids we could have a reduction by two orders of magnitude, namely, $\ell \sim 0.1 \mu \mathrm{m}$. Considering the confinement length to be $h \sim 10-100 \mu \mathrm{m}$, we find that it is very easy to realize a sufficiently dilute experimental system, which is in the strong coupling limit. While the laser intensity provides a continuous tuning parameter, the presence or absence of self-propulsion could move the system much faster in the parameter space. We note that Eq. (5) could be used to study the time dependence of the nonlinear dynamics of the colloids as in the analogous electrokinetic system [18].

The are a number of effects that we have not considered in the present analysis. We have neglected the temperature dependence of $D_{\text {eff }}$ in the calculation that led to Eq. (6), which could introduce corrections of the order of $\Delta T / T$ to the argument of the exponential. Moreover, hydrodynamic interactions have been shown to lead to nonlocal relations between the temperature profile and the diffusion coefficient of tracer particles [19]. However, we do not expect these effects to change the qualitative behavior of the system. We have also neglected the hydrodynamic interaction between the colloidal particles themselves. This is justified, as self-thermophoretic col- loids are effectively source dipoles and lead to velocity fields that decay as $1 / r^{3}$, which is faster than the thermophoretic interaction that decays as $1 / r^{2}$.

In conclusion, we have studied the collective behavior of active colloids that act as mobile heat sources, and found that thermo-repulsive colloids could organize into hollow bands, tubes, or shells, depending on the geometry, while thermo-attractive colloids could go unstable. We note that similar equations could be used to study collective chemotaxis of diffusiophoretically active particles using the analogy of nonequilibrium phoretic phenomena [7].

This work was supported by EPSRC.

* ramin.golestanian@physics.ox.ac.uk

[1] C. Ludwig, Sitz. ber. Akad. Wiss. Wien Math.-Nat. wiss. Kl 20, 539 (1856); C. Soret, Arch. Geneve 3, 48 (1879).

[2] R. Piazza and A. Guarino, Phys. Rev. Lett. 88, 208302 (2002); J. Lenglet et al., Phys. Rev. E 65, 031408 (2002); S. Duhr and D. Braun, Proc. Natl. Acad. Sci. U.S.A. 103, 19678 (2006); C. Debuschewitz and W. Köhler, Phys. Rev. Lett. 87, 055901 (2001); S. Wiegand, J. Phys.: Condens. Matter 16, R357 (2004); S.A. Putnam et al., Langmuir 23, 9221 (2007).

[3] S.R. de Groot and P. Mazur, Non-Equilibrium Thermodynamics (Dover, New York, 1984).

[4] F. Brochard and P.-G. de Gennes, C.R. Acad. Sci. Paris, Serie II 293, 1025 (1981); S. Fayolle et al., Phys. Rev. Lett. 95, 208301 (2005); J.K.G. Dhont et al., Langmuir 23, 1674 (2007); A. Würger, Phys. Rev. Lett. 98, 138301 (2007); R.D. Astumian, Proc. Nat. Acad. Sci. USA 104, 3 (2007); S. N. Rasuli and R. Golestanian, Phys. Rev. Lett. 101, 108301 (2008); A. Würger, Phys. Rev. Lett. 101, 108302 (2008).

[5] D. Braun and A. Libchaber, Phys. Rev. Lett. 89, 188103 (2002); H.-R. Jiang et al., Phys. Rev. Lett. 102, 208301 (2009).

[6] R. Golestanian, T. B. Liverpool, and A. Ajdari, Phys. Rev. Lett. 94, 220801 (2005); J.R. Howse et al., Phys. Rev. Lett. 99, 048102 (2007).

[7] R. Golestanian, T.B. Liverpool, and A. Ajdari, New J. Phys. 9, 126 (2007).

[8] H.-R. Jiang, N. Yoshinaga, and M. Sano, Phys. Rev. Lett. 105, 268302 (2010).

[9] D. Rings et al., Phys. Rev. Lett. 105, 090604 (2010).

[10] For simplicity, we have ignored the difference between the thermal conductivity of water and the colloid.

[11] R. Golestanian, Phys. Rev. Lett. 102, 188305 (2009).

[12] J. Palacci et al., Phys. Rev. Lett. 105, 088304 (2010).

[13] M. Enculescu and H. Stark, Phys. Rev. Lett. 107, 058301 (2011).

[14] Y. Levin, Rep. Prog. Phys. 65, 1577 (2002).

[15] H.-K. Tsao, J. Phys. Chem. B 102, 10243 (1998).

[16] L.D. Landau and E.M. Lifshitz, Fluid Mechanics 2nd edition (Pergamon, Oxford, England, 1987).

[17] K. Nomoto, Astrophys. J. 253, 798 (1982).

[18] R. Golestanian, Europhys. Lett. 52, 47 (2000).

[19] R. Golestanian and A. Ajdari, Europhys. Lett. 59, 800 (2002). 